\title{
Analytic high-order Douglas-Kroll-Hess electric field gradients
}

\author{
Remigius Mastalerz \\ Laboratorium für Physikalische Chemie, ETH Zurich, Hönggerberg Campus, Wolfgang-Pauli Strasse 10, \\ CH-8093 Zurich, Switzerland
}

\author{
Giampaolo Barone \\ Dipartimento di Chimica Inorganica e Analitica, Università di Palermo, Vialle delle Scienze, \\ Parco d'Orleans II, 90128 Palermo, Italy \\ Roland Lindh ${ }^{\text {a),b) }}$ \\ Department of Chemical Physics, Lund University, P.O. Box 124, 22100 Lund, Sweden \\ Markus Reiher ${ }^{\mathrm{a}), \mathrm{c})}$ \\ Laboratorium für Physikalische Chemie, ETH Zurich, Hönggerberg Campus, Wolfgang-Pauli Strasse 10, \\ CH-8093 Zurich, Switzerland
}

(Received 26 April 2007; accepted 26 June 2007; published online 20 August 2007)

In this work we present a comprehensive study of analytical electric field gradients in hydrogen halides calculated within the high-order Douglas-Kroll-Hess (DKH) scalar-relativistic approach taking picture-change effects analytically into account. We demonstrate the technical feasibility and reliability of a high-order DKH unitary transformation for the property integrals. The convergence behavior of the DKH property expansion is discussed close to the basis set limit and conditions ensuring picture-change-corrected results are determined. Numerical results are presented, which show that the DKH property expansion converges rapidly toward the reference values provided by four-component methods. This shows that in closed-shell cases, the scalar-relativistic $\operatorname{DKH}(2,2)$ approach which is of second order in the external potential for both orbitals and property operator yields a remarkable accuracy. As a parameter-dependence-free high-order DKH model, we recommend $\mathrm{DKH}(4,3)$. Moreover, the effect of a finite-nucleus model, different parametrization schemes for the unitary matrices, and the reliability of standard basis sets are investigated. (C) 2007 American Institute of Physics. [DOI: 10.1063/1.2761880]

\section{INTRODUCTION}

In recent years much effort has been devoted to the development of efficient and accurate relativistic schemes for the investigation of molecular properties. ${ }^{1,2}$ In particular, the electric field gradient (EFG) has received special attention as a property with $1 / r^{3}$ behavior, which can be calculated within first-order perturbation (i.e., linear response) theory. In the case of molecules containing heavy atoms, a correct calculation of molecular properties requires a consistent treatment of the property operator in the relativistic framework chosen.

A large variety of relativistic all-electron quantum chemical methods is available, ranging from highly accurate, but computationally expensive four-component approaches to elimination and transformation techniques. ${ }^{1,2}$ Among the latter the scalar-relativistic variant of the Douglas-Kroll-Hess $(\mathrm{DKH})$, unitary transformation theory ${ }^{3-6}$ has gained increasing attention during the past few years, leading to a rapid development of higher-order and even infinite-order Hamiltonians. ${ }^{5-10}$ But a naive calculation of molecular properties within the DKH framework using the nonrelativ-

\footnotetext{
a) Author to whom correspondence should be addressed.

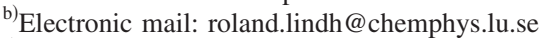

${ }^{c}$ Electronic mail: markus.reiher@phys.chem.ethz.ch
}

istic expression for the property operator is plagued by an artifact called picture-change effect. ${ }^{11-16}$

Recent second-order picture-change-affected DKH studies on tin compounds, for instance, revealed that following the estimates of Kellö and Sadlej ${ }^{12}$ for the iodine nucleus in $\mathrm{HI}$, one has to expect an overshoot of about $9 \%$ when calculating the electric field gradient from nonrelativistic property integrals. ${ }^{17}$ Pioneering attempts to cope with that problem gave birth to the development of numerical finite difference schemes. ${ }^{18,19}$ If the role of DKH orders larger than one shall be investigated for the transformed property operator, numerical methods suffer from inaccuracies due to the dependence of the calculated EFG on the displacement of model charges. A first step toward a fully analytical treatment of the property transformation has been taken by Malkin et al. by implementing a second-order property transformation. ${ }^{20} \mathrm{~A}$ detailed recent EFG study on the hydrogen halides has also been presented by Neese $e t$ al. instrumentalizing two different transformation schemes denoted as "forward" and "back" transformations. ${ }^{21}$ However, up to now no comprehensive study comprising high-DKH-order orbitals in combination with high-order DKH-transformed EFG operators has been presented in a purely analytical scheme. Hence, the goal of this work is to study the contribution of higher-order terms in the DKH-transformed electric field gradient close to the basis set limit. Then we determine the conditions which guarantee 
picture-change-corrected results in practice, for which we need to consider the balance of basis set size and DKH order of the property operator. Moreover, we discuss finite nuclear size effects in this work and compare the impact of different DKH unitary matrix parametrizations on the EFG. Fully relativistic four-component calculations of the EFG provide a point of reference in order to assess the accuracy of our one-component approach. Linear molecules are ideally suited for such a study, where large decontracted basis sets approaching the basis set limit can be employed. Therefore, we calculate the electric field gradient on the halogen nucleus in the series $\mathrm{HX}(\mathrm{X}=\mathrm{F}, \mathrm{Cl}, \mathrm{Br}, \mathrm{I}, \mathrm{At})$, which has already been discussed in the literature and thus offers an excellent opportunity for comparison. In a subsequent study we will focus on general molecules of extended structure.

This work is organized as follows. In Sec. II a brief account of the underlying theory of the DKH property transformation is presented. The subsequent Secs. III and IV comprise the computational methodology and details concerning the basis set construction, respectively. The following Secs. $\mathrm{V}$ and VI contain a discussion of results and concluding remarks, respectively.

\section{THEORETICAL BACKGROUND}

Starting from the four-component one-electron Dirac picture, an electric-field-like property operator $X$ is added to the original Dirac-Hamiltonian $H_{D}$,

$$
H_{D}(\lambda)=H_{D}+\lambda X
$$

as a perturbation in linear response theory. $H_{D}$ denotes the unperturbed one-electron Dirac-Hamiltonian in standard notation,

$$
H_{D}=c \boldsymbol{\alpha} \cdot \boldsymbol{p}+\left(\beta-\mathbf{1}_{4}\right) m c^{2}+V \mathbf{1}_{4},
$$

and $\lambda$ is a formal expansion parameter. The natural constants $c$ and $m$ in Eq. (2) are the speed of light and the rest mass of the electron, respectively. The linear momentum operator $\boldsymbol{p}$ is given in its well-known differential form, $\beta$ is a $(4 \times 4)$ diagonal Dirac matrix with $(1,1,-1,-1)$ as entries, and $\boldsymbol{\alpha}$ denotes a 3 -vector, whose elements consist of $(4 \times 4)$ matrices constructed from the Pauli spin matrices. ${ }^{22}$ The external potential $V$ represents the instantaneous Coulombic electronnucleus interaction. In accordance with Eq. (1), the relativistic energy $E_{D}$ of the perturbed system is given by

$$
E_{D}(\lambda)=\left\langle\Psi_{D}(\lambda)\left|H_{D}(\lambda)\right| \Psi_{D}(\lambda)\right\rangle,
$$

with $\Psi_{D}(\lambda)$ representing the four-component wave function in the presence of the additional potential energy term $\lambda X$. An electric-field-dependent property is described fully by an even and thus block-diagonal operator, ${ }^{23}$

$$
X=\left(\begin{array}{cc}
X_{L L} & 0 \\
0 & X_{S S}
\end{array}\right)
$$

In the case of an EFG calculation $X$ features a completely diagonal form. However, a solution of Eq. (3) is not mandatory, and linear response theory may be used instead. Taylor series expansions for energy and wave function usually converge fast in the case of weak perturbations. It is hence suf- ficient to consider the lowest order correction $E_{D}^{(1)}$ to the unperturbed energy $E_{D}(\lambda=0)$,

$$
E_{D}^{(1)}=\left[\frac{d E_{D}(\lambda)}{d \lambda}\right]_{\lambda=0}=\left\langle\Psi_{D}^{(\lambda=0)}|X| \Psi_{D}^{(\lambda=0)}\right\rangle .
$$

Hence, the unperturbed wave function is sufficient in a fourcomponent (and consequently also in a two-component) framework for the calculation of linear response quantities. Note that Eq. (5) requires that the wave function employed fulfills the Hellmann-Feynman theorem.

In a quasirelativistic scheme, we block diagonalize the unperturbed Dirac-Hamiltonian $H_{D}$ by a suitably chosen unitary transformation $U$,

$$
H_{b d}=U H_{D} U^{\dagger}=\left(\begin{array}{cc}
h_{+} & 0 \\
0 & h_{-}
\end{array}\right)=\sum_{k=0}^{\infty} \mathcal{E}_{k},
$$

yielding a $(2 \times 2)$ Hamiltonian $h_{+}$that describes only electronic states of the positive-energy spectrum (note that this notation includes the electronic bound states). In DKH theory, $U$ is built up by a sequence of infinitely many unitary matrices $U_{m}$. Each unitary matrix $U_{m}$ is expanded in terms of a power series of the parameter $W_{m}$ according to the most general ansatz,

$$
U_{m}=\sum_{j=0}^{\infty} a_{m, j} W_{m}^{j}=1+\sum_{j=1}^{\infty} a_{m, j} W_{m}^{j} .
$$

The expansion parameter $W_{m}$ has to be anti-Hermitian and is chosen such that the off-diagonal blocks are diminished order by order in the external potential. The set of expansion coefficients $\left\{a_{m, j}\right\}$ must guarantee unitarity of $U_{m}$. The socalled optimum parametrization given in Ref. 5 allows us to provide a set of expansion coefficients $\left\{a_{m, j}\right\}$, which yields truncated unitary transformations that fulfill the condition of unitarity as close as possible upon truncation of the series in Eq. (7) after a given order. Although it is not a crucial part of DKH theory, several sets of expansion coefficients $\left\{a_{m, j}\right\}$ were devised, among them the square-root parametrization first introduced by Douglas and Kroll. ${ }^{3}$

The transformation $U$ must also be applied to the unperturbed four-component wave function,

$$
U \Psi_{D}^{(\lambda=0)}=U\left(\begin{array}{c}
\Psi_{L} \\
\Psi_{S}
\end{array}\right)=\left(\begin{array}{c}
\Phi_{L} \\
0
\end{array}\right),
$$

eliminating its small-component part $\Psi_{S}$ of the 4-spinor and yielding the exact two-component DKH wave function $\Phi_{L}$. Taking into account the first-order correction to the energy given by Eq. (5), which requires exclusively the unperturbed wave function, we obtain

$$
E_{D}^{(1)}=\left\langle U \Psi_{D}^{(\lambda=0)}\left|U X U^{\dagger}\right| U \Psi_{D}^{(\lambda=0)}\right\rangle=\left\langle\Phi_{L}\left|X_{\mathrm{DKH} \infty}^{L L}\right| \Phi_{L}\right\rangle .
$$

So far Eq. (9) does not contain any approximation. In practice, however, DKH transformations are truncated with respect to a predefined order in the external potential, resulting in a systematic approximation hierarchy for the desired expectation value, 


$$
\langle X(n, m)\rangle=\left\langle\Phi_{L}^{\mathrm{DKH} n}\left|\sum_{k=0}^{m} X_{\mathcal{E}, k}\right| \Phi_{L}^{\mathrm{DKH} n}\right\rangle,
$$

where $n$ and $m$ refer to the DKH order of the orbitals (determined from an $n$th order Hamiltonian) and property operator, respectively.

The procedure for the calculation of electric-fielddependent DKH properties discussed so far was demonstrated within a one-component formalism for radial momenta as property operators. ${ }^{24,25}$ We may note that Malkin et al. applied a similar low-order scheme for the calculation of magnetic properties which they called back transformation. ${ }^{26}$ We should, however, note that these authors actually did not use a truly four-component framework in this case, but actually considered the transformation of the four-component property operator. ${ }^{27}$ Nonetheless, it is in principle possible $e^{23}$ to return to the original Dirac picture starting from Eq. (8), in which the DKH-transformed unperturbed two-component wave function is obtained, by applying the inverse transformation,

$$
U^{-1}\left(\begin{array}{c}
\Phi_{L} \\
0
\end{array}\right)=\left(\begin{array}{c}
\Psi_{L} \\
\Psi_{S}
\end{array}\right)
$$

According to this ansatz the evaluation of the expectation value $\langle X\rangle$ then takes place in the original four-component framework. The advantage of this procedure is that it is an intrinsically picture-change-free formalism. Within a standard DKH program, which follows Hess' original implementation, ${ }^{4}$ this alternative cannot be implemented as no explicit representation of $U$, which would require the introduction of a small-component basis in one way or another, is available.

One should not confuse this particular four-component approach with the term "back transformation" as used in Ref. 21 , referring to a general application of perturbationindependent unitary transformations $U_{m}$. Following Neese et al., ${ }^{21}$ a "forward transformation," however, would feature an explicit dependence of the unitary transformations $U_{m}(\lambda)$ on the perturbation strength $\lambda$. As both procedures involve unitary transformations, they must be identical at infinite order. At low order, however, they may deviate. We should stress that we consider perturbation-independent unitary matrices, $U_{m}(\lambda=0)$, i.e., those called back transformation matrices in Ref. 21, as the proper choice as these would exactly produce the unperturbed wave function of the fourcomponent approach, as required by Eq. (5). ${ }^{23}$ The preceding considerations reflect a general treatment of electric-fieldlike properties within the DKH framework, which has been explicated in detail in Ref. 23. We now focus on the calculation of picture-change-corrected electric field gradients. The perturbing one-electron operator, which accounts for the electronic contribution to the EFG at nucleus $A$, is given by

$$
X_{A, \mu \nu}=\sum_{i} \frac{3 r_{i A, \mu} r_{i A, \nu}-\boldsymbol{r}_{i A}^{2} \delta_{\mu \nu}}{\left|\boldsymbol{r}_{i A}\right|^{5}},
$$

where $\boldsymbol{r}_{i A}=\boldsymbol{r}_{i}-\boldsymbol{R}_{A}$ defines the position of electron $i$ relative to nucleus $A$. The calculation of the nuclear contribution to the $\mathrm{EFG}$, which is skipped in Eq. (12), is trivial in the regime of the Born-Oppenheimer approximation, as it merely yields an additive constant.

Note that all what has been discussed so far is valid for the one-electron case. It is straightforward to generalize all statements for the $N$-electron case, where the one-electron perturbation operator enters the sum of $N$ such one-electron operators. Accordingly, all what has been said for the oneelectron wave function transfers to molecular spinors and orbitals, which set up the many-electron wave function. The scalar-relativistic, i.e., one-component, expressions of the DKH-transformed property operator in basis set representation are obtained after separation and neglect of all spindependent terms. ${ }^{24}$

\section{COMPUTATIONAL DETAILS}

All scalar-relativistic calculations were carried out with the MOLCAS electronic structure package, ${ }^{28}$ into which we implemented our arbitrary-order DKH property module. ${ }^{24}$ The bond distances of the hydrogen halides series were taken from Ref. 21 and are given in bohrs as follows (we use Hartree atomic units throughout): $\mathrm{HF}: 1.732549, \mathrm{HCl}$ : 2.408 502, HBr: 2.672 946, HI: 3.040798 , and HAt: 3.250392 .

The accurate calculation of the EFG requires large decontracted basis sets which is a sensitive issue. Note that many previous papers did either not provide the full list of exponents but a rather vague description of the quality of the basis set or recursively refer to rather old papers which makes it difficult to reproduce the results published. Therefore, the construction of the basis sets used in this work is described in detail in Sec. IV. In particular, we provide all exponents.

The overall basis set sizes used in the benchmark calculations are At $(37 s / 32 p / 22 d / 15 f / 4 g)$, I $(35 s / 28 p / 19 d / 8 f / 4 g), \quad \mathrm{Br} \quad(30 s / 22 p / 14 d / 8 f / 3 g), \quad \mathrm{Cl}$ $(24 s / 19 p / 7 d / 5 f), \mathrm{F}(23 s / 17 p / 6 d / 4 f)$, and $\mathrm{H}(20 s / 5 p / 4 d)$. Tight convergence criteria were imposed on the calculation of the wave function. The maximum allowed difference in total energy was set to $10^{-10}$ hartree, and the maximally allowed changes in the density matrix elements and Fock matrix elements were chosen to be $10^{-6}$ and $0.5 \times 10^{-7}$ a.u., respectively. The norm of the orbital displacement vector used in the $\mathrm{C}^{2}$-DIIS procedure was fixed to $0.2 \times 10^{-7}$. The Dirac-Hartree-Fock calculations were performed with the DIRAC (Ref. 29) program package using the same bond distances, basis sets, and finite-nucleus exponents as in the onecomponent calculations. The small-component exponents were obtained from applying the atomic kinetic balance condition to the large component basis set and using both the upward and downward generated derivatives.

\section{BASIS SET CONSTRUCTION AND BASIS SET DEPENDENCE OF THE EFG}

The basis sets already introduced in Sec. III were primarily generated to yield an accurate EFG close to the limit within the approximations involved, i.e., under neglect of spin-orbit coupling and using a single Slater determinant as an approximation for the wave function. They were not fully 
TABLE I. $s$ and $p$ exponents of the decontracted basis sets used in all nonrelativistic, DKH, and four-component calculations.

\begin{tabular}{|c|c|c|c|c|c|}
\hline At & I & $\mathrm{Br}$ & $\mathrm{Cl}$ & F & $\mathrm{H}$ \\
\hline \multicolumn{6}{|c|}{$s$ exponents } \\
\hline $1.0143072 E+09$ & $3.0239251 E+08$ & $1.0041516 E+08$ & $3.1024400 E+07$ & $2.1267360 E+05$ & $1.1629377 E+05$ \\
\hline $2.4150171 E+08$ & $7.7536542 E+07$ & $2.6683516 E+07$ & $1.4102000 E+07$ & $7.0891200 E+04$ & $4.1865759 E+04$ \\
\hline $6.0375427 E+07$ & $2.0605227 E+07$ & $9.1612279 E+06$ & $6.4100000 E+06$ & $2.5318287 E+04$ & $1.2686593 E+04$ \\
\hline $1.6075673 E+07$ & $7.0496680 E+06$ & $3.5487189 E+06$ & $9.5960000 E+05$ & $9.7378027 E+03$ & $4.2288645 E+03$ \\
\hline $5.5075645 E+06$ & $2.6847677 E+06$ & $1.5263923 E+06$ & $2.1830000 E+05$ & $4.0574178 E+03$ & $1.5103087 E+03$ \\
\hline $2.1004143 E+06$ & $1.1129577 E+06$ & $6.9365692 E+05$ & $6.1810000 E+04$ & $1.6905907 E+03$ & $5.8088799 E+02$ \\
\hline $8.7917001 E+05$ & $4.8541403 E+05$ & $3.2121659 E+05$ & $2.0140000 E+04$ & $7.6845034 E+02$ & $2.5255999 E+02$ \\
\hline $3.9045618 E+05$ & $2.2082655 E+05$ & $1.4934913 E+05$ & $7.2640000 E+03$ & $3.6592873 E+02$ & $1.1479999 E+02$ \\
\hline $1.8273552 E+05$ & $1.0378765 E+05$ & $7.0198343 E+04$ & $2.8320000 E+03$ & $1.8296437 E+02$ & $5.4666666 E+01$ \\
\hline $8.8838178 E+04$ & $5.0202359 E+04$ & $3.3572148 E+04$ & $1.1750000 E+03$ & $9.6297035 E+01$ & $2.7333332 E+01$ \\
\hline $4.4616106 E+04$ & $2.4901714 E+04$ & $1.6392455 E+04$ & $5.1260000 E+02$ & $5.3498353 E+01$ & $1.4385964 E+01$ \\
\hline $2.2979252 E+04$ & $1.2646252 E+04$ & $8.1711624 E+03$ & $2.3300000 E+02$ & $3.0570487 E+01$ & $8.4623317 E+00$ \\
\hline $1.2095138 E+04$ & $6.5646460 E+03$ & $4.1536224 E+03$ & $1.0950000 E+02$ & $1.6983604 E+01$ & $5.2889573 E+00$ \\
\hline $6.4907973 E+03$ & $3.4777873 E+03$ & $2.1510030 E+03$ & $5.2860000 E+01$ & $9.9903553 E+00$ & $3.1111513 E+00$ \\
\hline $3.5524824 E+03$ & $1.8783840 E+03$ & $1.1336020 E+03$ & $2.5840000 E+01$ & $5.8766796 E+00$ & $1.6374480 E+00$ \\
\hline $1.9845589 E+03$ & $1.0342444 E+03$ & $6.0716239 E+02$ & $1.2170000 E+01$ & $3.4568703 E+00$ & $8.6181478 E-01$ \\
\hline $1.1323731 E+03$ & $5.8126033 E+02$ & $3.2997916 E+02$ & $6.0300000 E+00$ & $2.0334531 E+00$ & $4.5358673 E-01$ \\
\hline $6.6017267 E+02$ & $3.3467185 E+02$ & $1.8148940 E+02$ & $3.0120000 E+00$ & $1.1961489 E+00$ & $2.3872985 E-01$ \\
\hline $3.9392589 E+02$ & $2.0023231 E+02$ & $1.0042325 E+02$ & $1.5110000 E+00$ & $6.4656697 E-01$ & $1.2564729 E-01$ \\
\hline $2.4021531 E+02$ & $1.3363760 E+02$ & $5.4956308 E+01$ & $6.6040000 E-01$ & $3.5920387 E-01$ & $6.9804000 E-02$ \\
\hline $1.4900652 E+02$ & $8.8642082 E+01$ & $3.0807026 E+01$ & $2.9260000 E-01$ & $1.9955770 E-01$ & \\
\hline $9.4000919 E+01$ & $5.5489724 E+01$ & $1.7391737 E+01$ & $1.2540000 E-01$ & $9.9778850 E-02$ & \\
\hline $5.9699821 E+01$ & $3.3718898 E+01$ & $9.7579856 E+00$ & $6.2700000 E-02$ & $4.9889425 E-02$ & \\
\hline $3.7696078 E+01$ & $2.0294880 E+01$ & $5.2575835 E+00$ & $3.1350000 E-02$ & & \\
\hline $2.3386529 E+01$ & $1.2576413 E+01$ & $2.8683063 E+00$ & & & \\
\hline $1.5284999 E+01$ & $7.9259000 E+00$ & $1.5495118 E+00$ & & & \\
\hline $9.4028096 E+00$ & $4.9928561 E+00$ & $7.4794702 E-01$ & & & \\
\hline $5.9292233 E+00$ & $3.0482607 E+00$ & $3.8470540 E-01$ & & & \\
\hline $3.8706010 E+00$ & $1.8314611 E+00$ & $1.8885606 E-01$ & & & \\
\hline $2.2177953 E+00$ & $1.0586577 E+00$ & $8.9337860 E-02$ & & & \\
\hline $1.3405729 E+00$ & $5.5942026 E-01$ & & & & \\
\hline $8.4309331 E-01$ & $2.9653867 E-01$ & & & & \\
\hline $5.6531041 E-01$ & $1.5000804 E-01$ & & & & \\
\hline $2.9318475 E-01$ & $7.2609908 E-02$ & & & & \\
\hline $1.4823369 E-01$ & $3.2097135 E-02$ & & & & \\
\hline \multirow{2}{*}{\multicolumn{6}{|c|}{$\begin{array}{l}7.0506504 E-02 \\
2.7470882 E-02\end{array}$}} \\
\hline & & & & & \\
\hline \multicolumn{6}{|c|}{$p$ exponents } \\
\hline $7.2251407 E+07$ & $4.4437221 E+07$ & $3.1536694 E+06$ & $2.8889190 E+05$ & $2.6342910 E+03$ & $4.3950000 E+00$ \\
\hline $2.4932854 E+07$ & $1.0316962 E+07$ & $4.6584298 E+05$ & $1.3131450 E+05$ & $1.1453439 E+03$ & $1.9950000 E+00$ \\
\hline $8.8937376 E+06$ & $2.8276690 E+06$ & $9.9999180 E+04$ & $5.9688400 E+04$ & $5.2061087 E+02$ & $9.0600000 E-01$ \\
\hline $3.3454100 E+06$ & $8.6513173 E+05$ & $2.6943107 E+04$ & $2.7131104 E+04$ & $2.2635255 E+02$ & $4.1100000 E-01$ \\
\hline $1.3140444 E+06$ & $2.8739819 E+05$ & $8.6599605 E+03$ & $1.2332320 E+04$ & $1.0778692 E+02$ & $1.8660000 E-01$ \\
\hline $5.3608132 E+05$ & $1.0268966 E+05$ & $3.1997314 E+03$ & $5.6056000 E+03$ & $5.3893464 E+01$ & \\
\hline $2.2603474 E+05$ & $3.9211761 E+04$ & $1.3148600 E+03$ & $2.4800000 E+03$ & $2.8364981 E+01$ & \\
\hline $9.8286709 E+04$ & $1.5955056 E+04$ & $5.8516637 E+02$ & $6.0370000 E+02$ & $1.5758323 E+01$ & \\
\hline $4.4052614 E+04$ & $6.9078934 E+03$ & $2.7666970 E+02$ & $1.9560000 E+02$ & $9.2696017 E+00$ & \\
\hline $2.0355617 E+04$ & $3.1680314 E+03$ & $1.3713201 E+02$ & $7.4150000 E+01$ & $5.1497787 E+00$ & \\
\hline $9.7031233 E+03$ & $1.5274574 E+03$ & $7.0358707 E+01$ & $3.0940000 E+01$ & $2.8609881 E+00$ & \\
\hline $4.7758375 E+03$ & $7.6826049 E+02$ & $3.7014740 E+01$ & $1.3690000 E+01$ & $1.5057832 E+00$ & \\
\hline $2.4266900 E+03$ & $4.0029857 E+02$ & $1.9815980 E+01$ & $6.2290000 E+00$ & $8.3654622 E-01$ & \\
\hline $1.2710526 E+03$ & $2.1442158 E+02$ & $1.0599489 E+01$ & $2.8780000 E+00$ & $4.6474790 E-01$ & \\
\hline $6.8407924 E+02$ & $1.1729112 E+02$ & $5.5846938 E+00$ & $1.2820000 E+00$ & $2.4460415 E-01$ & \\
\hline $3.7693166 E+02$ & $6.5249304 E+01$ & $2.9206897 E+00$ & $5.6410000 E-01$ & $1.2873903 E-01$ & \\
\hline $2.1194901 E+02$ & $3.6524103 E+01$ & $1.5099621 E+00$ & $2.3480000 E-01$ & $6.6020015 E-02$ & \\
\hline $1.2075749 E+02$ & $2.0313426 E+01$ & $7.1682232 E-01$ & $9.3120000 E-02$ & & \\
\hline $6.9304384 E+01$ & $1.1480568 E+01$ & $3.3634819 E-01$ & $4.6556000 E-02$ & & \\
\hline $4.0524414 E+01$ & $6.4884393 E+00$ & $1.5263369 E-01$ & & & \\
\hline $2.3598368 E+01$ & $3.6011336 E+00$ & $6.6724350 E-02$ & & & \\
\hline
\end{tabular}


TABLE I. (Continued.)

\begin{tabular}{ccccc}
\hline \hline At & $\mathrm{I}$ & $\mathrm{Br}$ & $\mathrm{Cl}$ & $\mathrm{F}$ \\
\hline $1.3497835 E+01$ & $1.9589803 E+00$ & $2.7386094 E-02$ & & \\
$7.8076208 E+00$ & $1.0466017 E+00$ & & \\
$4.4667435 E+00$ & $5.1503900 E-01$ & & \\
$2.5211716 E+00$ & $2.5018062 E-01$ & & \\
$1.3993280 E+00$ & $1.1740677 E-01$ & & \\
$7.5966311 E-01$ & $5.3158403 E-02$ & & \\
$3.9017758 E-01$ & $2.2475771 E-02$ & & \\
$1.9315865 E-01$ & & & \\
$9.3229402 E-02$ & & & \\
$4.3409155 E-02$ & & & \\
$1.8420797 E-02$ & & & \\
\hline \hline
\end{tabular}

optimized with respect to the electronic energy, but they perform better with respect to the energy and EFG than the basis sets used in previous studies ${ }^{21,30}$ on the halogen series.

As already mentioned, the basis set information was not fully provided in previous work by other groups. For this reason we explicitly give all exponents of our basis sets of primitive Gaussians in Tables I and II.

The optimization of the basis sets was carried out by successively extending a primary basis set. Additional exponents were incorporated until the change of the EFG was below a predefined tight threshold and convergence was ascertained. This procedure, thus, corresponds to an individual optimization of additional exponents. In the case of the heavier elements $(\mathrm{Br}, \mathrm{I}, \mathrm{At})$, we chose to take the $s, p, d$, and $f$ exponents (including the diffuse functions) from the relativistic quadruple-zeta basis sets devised by Dyall ${ }^{31}$ as starting point. For At the extension comprised two tight $s$ exponents and four $g$ functions derived from a geometric progression. The iodine basis set was augmented by one tight $s$ function, the valence and core correlating $f$ functions of the original basis set, two additional tight $f$ functions, and four $g$ functions consisting of the same exponents as in the case of At. For Br the valence and core correlating $f$ functions of the original basis set, two additional tight $f$ functions, and three $g$ functions were appended. The $\mathrm{Cl}$ basis set was generated from the cc-pV5Z basis set ${ }^{32}$ by supplementing the original exponents by two tight and two diffuse $s$ functions, six tight and one diffuse $p$ functions, three steep $d$ functions, and finally two steep $f$ functions. The $s$ and $p$ functions of the $\mathrm{F}$ basis set were generated by using an increasing ratio of subsequent exponents ranging from 2 to 3 in the case of the $s$ functions and from 1.95 to 2.3 in the case of $p$ functions. The $d$ and $f$ exponents were taken from the standard cc-pV5Z basis set and extended by two additional tight $d$ functions and one steep $f$ exponent. The $s$ exponents of the $\mathrm{H}$ basis set were generated in the same fashion using a ratio ranging from 1.8 to 2.78 , and the $p$ and $d$ exponents were provided by a cc-pV6Z (Ref. 33) basis set. It is noteworthy that the EFG depends to a large extent on steep higher angular momentum exponents centered at the nucleus the EFG calculation is performed on. Diffuse exponents of the same angular momentum, however, practically do not contribute to the EFG. This can be understood by considering the fact that the EFG operator features a $r^{-3}$ dependence, where $r$ denotes the electron-nucleus distance, and hence probes the core region. Nevertheless diffuse functions on nearby atoms may contribute to the electric field gradient calculated at a specific nucleus, which has to be considered when investigating extended molecules.

\section{RESULTS AND DISCUSSION}

\section{A. Convergence of the DKH electric field gradient}

The results of the scalar-relativistic DKH-Hartree-Fock and the Dirac-Hartree-Fock (DHF) calculation are presented in Tables III-VII.

\section{Light homologues: $\mathrm{HF}, \mathrm{HCl}$}

In the case of the lighter elements relativistic effects are, as expected, negligible. A nonrelativistic EFG calculation on $\mathrm{HF}$ recovers already $99.7 \%$ of the $\operatorname{DKH}(7,7)$ value and $99.6 \%$ of the four-component DHF treatment, respectively. A $\operatorname{DKH}(2,2)$ calculation reproduces the four-component DHF reference within a deviation of $5 \times 10^{-4}$ a.u. The absolute picture-change error (PCE), approximated by the difference of the $\operatorname{DKH}(7,0)$ and $\operatorname{DKH}(7,7)$ expectation values according to the following formula

$$
\operatorname{PCE}\left(q_{z z}\right)=q_{z z}(7,0)-q_{z z}(7,7)
$$

comprises less than $5 \times 10^{-3}$ a.u. The relative picture-change error estimated from the ratio

$$
\operatorname{PCE}_{\mathrm{rel}}\left(q_{z z}\right)=\left(\frac{q_{z z}(7,0)}{q_{z z}(7,7)} \times 100 \%\right)-100 \%
$$

is found to be smaller than $0.2 \%$ in the case of HF.

A nonrelativistic calculation of the EFG at the $\mathrm{Cl}$ nucleus in $\mathrm{HCl}$ covers about $99.6 \%$ of the $\operatorname{DKH}(7,7)$ and DHF reference values. A DKH $(2,2)$ calculation of the EFG shows an accuracy of $1.1 \cdot 10^{-3}$ a.u. compared to a DIRAC DHF calculation. With an absolute value of 0.03 a.u. and a relative value of $0.8 \%$, the PCE is comparatively small.

\section{Heavy homologues: HBr, HI, HAt}

For the $\mathrm{Br}$ nucleus in $\mathrm{HBr}$ relativistic effects, however, begin to affect the expectation value of the EFG remarkably. Calculating the EFG in $\mathrm{HBr}$ from a nonrelativistic wave function reproduces merely about $93 \%$ of the $\operatorname{DKH}(7,7)$ 
TABLE II. $d, f$, and $g$ exponents of the decontracted basis sets used in all nonrelativistic, DKH, and four-component calculations.

\begin{tabular}{|c|c|c|c|c|c|}
\hline At & $\mathrm{I}$ & $\mathrm{Br}$ & $\mathrm{Cl}$ & $\mathrm{F}$ & $\mathrm{H}$ \\
\hline \multicolumn{6}{|c|}{$d$ exponents } \\
\hline $1.9034949 E+05$ & $2.4531944 E+04$ & $2.0716415 E+03$ & $1.8117000 E+02$ & $1.2633000 E+02$ & $4.9740000 E+00$ \\
\hline $4.4572260 E+04$ & $6.1561227 E+03$ & $5.8992661 E+02$ & $4.1175000 E+01$ & $2.8711999 E+01$ & $2.2150000 E+00$ \\
\hline $1.4282757 E+04$ & $2.1509140 E+03$ & $2.2300816 E+02$ & $1.2477300 E+01$ & $7.7600000 E+00$ & $9.8600000 E-01$ \\
\hline $5.4662239 E+03$ & $8.9178828 E+02$ & $9.7375981 E+01$ & $3.7810000 E+00$ & $3.0320000 E+00$ & $4.3900000 E-01$ \\
\hline $2.3620925 E+03$ & $4.1184308 E+02$ & $4.6183629 E+01$ & $1.5290000 E+00$ & $1.1850000 E+00$ & \\
\hline $1.1150297 E+03$ & $2.0485293 E+02$ & $2.3040574 E+01$ & $6.1800000 E+01$ & $4.6300000 E-01$ & \\
\hline $5.6183005 E+02$ & $1.0711474 E+02$ & $1.1897373 E+01$ & $2.5000000 E+01$ & & \\
\hline $2.9682816 E+02$ & $5.8128222 E+01$ & $6.2083631 E+00$ & & & \\
\hline $1.6269028 E+02$ & $3.2329802 E+01$ & $3.2342174 E+00$ & & & \\
\hline $9.1351283 E+01$ & $1.8214413 E+01$ & $1.6791374 E+00$ & & & \\
\hline $5.2299012 E+01$ & $1.0385388 E+01$ & $8.7026678 E-01$ & & & \\
\hline $3.0186267 E+01$ & $5.9043953 E+00$ & $4.5992947 E-01$ & & & \\
\hline $1.7352767 E+01$ & $3.2932275 E+00$ & $1.9525117 E-01$ & & & \\
\hline $9.9256123 E+00$ & $1.8147700 E+00$ & $7.4606071 E-02$ & & & \\
\hline $5.6533216 E+00$ & $1.0037929 E+00$ & & & & \\
\hline $3.1660082 E+00$ & $6.0503310 E-01$ & & & & \\
\hline $1.7309287 E+00$ & $3.7251689 E-01$ & & & & \\
\hline $9.5105066 E-01$ & $1.5580080 E-01$ & & & & \\
\hline $5.7159618 E-01$ & $6.0315220 E-02$ & & & & \\
\hline \multicolumn{6}{|l|}{$3.3761399 E-01$} \\
\hline \multicolumn{6}{|l|}{$1.3892468 E-01$} \\
\hline \multicolumn{6}{|l|}{$5.3547792 E-02$} \\
\hline \multicolumn{6}{|c|}{$f$ exponents } \\
\hline $2.9832111 E+03$ & $1.0972882 E+02$ & $4.2980103 E+02$ & $2.1912000 E+01$ & $1.9972599 E+01$ & \\
\hline $9.8834645 E+02$ & $2.8876006 E+01$ & $8.9541883 E+01$ & $4.9800000 E+00$ & $5.3980000 E+00$ & \\
\hline $4.1900451 E+02$ & $8.0211128 E+00$ & $2.0350428 E+01$ & $1.3450000 E+00$ & $2.0780000 E+00$ & \\
\hline $2.0048009 E+02$ & $3.1800844 E+00$ & $7.2306031 E+00$ & $6.5600000 E-01$ & $8.0000000 E-01$ & \\
\hline $1.0365833 E+02$ & $1.3044671 E+00$ & $2.6487448 E+00$ & $3.2000000 E-01$ & & \\
\hline $5.5917913 E+01$ & $5.8827498 E-01$ & $8.2694657 E-01$ & & & \\
\hline $3.1127876 E+01$ & $2.5020121 E-01$ & $3.4166286 E-01$ & & & \\
\hline $1.7542308 E+01$ & $1.4874551 E-01$ & $1.8900582 E-01$ & & & \\
\hline \multicolumn{6}{|l|}{$9.8863737 E+00$} \\
\hline \multicolumn{6}{|l|}{$5.5184395 E+00$} \\
\hline \multicolumn{6}{|l|}{$2.9904382 E+00$} \\
\hline \multicolumn{6}{|l|}{$1.5189767 E+00$} \\
\hline \multicolumn{6}{|l|}{$5.4776846 E-01$} \\
\hline \multicolumn{6}{|l|}{$2.3105119 E-01$} \\
\hline \multicolumn{6}{|l|}{$1.3142992 E-01$} \\
\hline \multicolumn{6}{|c|}{$g$ exponents } \\
\hline $1.0000000 E+00$ & $1.0000000 E+00$ & $1.0000000 E+00$ & & & \\
\hline $4.0000000 E+00$ & $4.0000000 E+00$ & $4.0000000 E+00$ & & & \\
\hline $1.6000000 E+01$ & $1.6000000 E+01$ & $1.6000000 E+01$ & & & \\
\hline $6.4000000 E+01$ & $6.4000000 E+01$ & & & & \\
\hline
\end{tabular}

value and DHF reference. The $\operatorname{DKH}(2,2)$ value differs only $4 \times 10^{-3}$ a.u. from the DHF reference. An absolute PCE of about 0.3 a.u. resulting in a relative effect of the order of $4 \%$, however, gives rise to larger deviations, when neglecting the property transformation.

For the two heaviest molecules HI and HAt in our study, the influence of relativity on the EFG is far greater than in the cases mentioned before. A nonrelativistic treatment of the property integrals in $\mathrm{HI}$ leads to a drop of the EFG by almost $17 \%$ when compared to the $\operatorname{DKH}(7,7)$ or the DHF calculation. In the case of HAt a nonrelativistic expression for the EFG underestimates the DHF result by about $41 \%$. The
$\operatorname{DKH}(2,2)$ approach yields compared to the DHF reference a deviation of 0.01 a.u. for $\mathrm{HI}$ and a deviation of 0.36 a.u. for HAt, respectively. The absolute PCE is about 1.24 a.u. in the case of HI, which increases to 9.90 a.u. for HAt, leading to a significant relative PCE of $11 \%$ for the EFG on the iodine nucleus, which rises to about $37 \%$ for HAt. An overview of the relative amount of scalar-relativistic effects and the relative size of the picture-change error for all molecules under investigation is given in Table VIII.

According to these results a relativistic treatment avoiding the PCE of the molecular property is mandatory for the heavier molecules ( $\mathrm{HBr}, \mathrm{HI}, \mathrm{HAt})$. As has already been 
TABLE III. Electronic energy and the principal component of the diagonalized electric field gradient tensor $\left(q_{z z}\right)$ in HF (in a.u.) calculated at the F nucleus.

\begin{tabular}{|c|c|c|c|c|c|c|c|c|c|}
\hline $\mathrm{DKH} n$ & Energy & $q_{z z}(n, 0)$ & $q_{z z}(n, 1)$ & $q_{z z}(n, 2)$ & $q_{z z}(n, 3)$ & $q_{z z}(n, 4)$ & $q_{z z}(n, 5)$ & $q_{z z}(n, 6)$ & $q_{z z}(n, 7)$ \\
\hline \multicolumn{10}{|c|}{ Point charge nucleus/Optimum parametrization } \\
\hline Nonrel & -100.07071071 & 2.799747 & & & & & & & \\
\hline DKH2 & -100.15749139 & 2.813983 & 2.809265 & 2.809265 & & & & & \\
\hline DKH3 & -100.15755959 & 2.813984 & 2.809265 & 2.809266 & 2.809266 & & & & \\
\hline DKH4 & -100.15755880 & 2.813984 & 2.809265 & 2.809266 & 2.809266 & 2.809266 & & & \\
\hline DKH5 & -100.15755882 & 2.813984 & 2.809265 & 2.809266 & 2.809266 & 2.809266 & 2.809266 & & \\
\hline DKH6 & -100.15755882 & 2.813984 & 2.809265 & 2.809266 & 2.809266 & 2.809266 & 2.809266 & 2.809266 & \\
\hline DKH7 & -100.15755882 & 2.813984 & 2.809265 & 2.809266 & 2.809266 & 2.809266 & 2.809266 & 2.809266 & 2.809266 \\
\hline \multicolumn{10}{|c|}{ Finite nucleus/Optimum parametrization } \\
\hline Nonrel & -100.07068738 & 2.799746 & & & & & & & \\
\hline DKH2 & -100.15746760 & 2.813983 & 2.809265 & 2.809265 & & & & & \\
\hline DKH3 & -100.15753577 & 2.813984 & 2.809265 & 2.809266 & 2.809266 & & & & \\
\hline DKH4 & -100.15753498 & 2.813984 & 2.809265 & 2.809266 & 2.809266 & 2.809266 & & & \\
\hline DKH5 & -100.15753500 & 2.813984 & 2.809265 & 2.809266 & 2.809266 & 2.809266 & 2.809266 & & \\
\hline DKH6 & -100.15753500 & 2.813984 & 2.809265 & 2.809266 & 2.809266 & 2.809266 & 2.809266 & 2.809266 & \\
\hline DKH7 & -100.15753500 & 2.813984 & 2.809265 & 2.809266 & 2.809266 & 2.809266 & 2.809266 & 2.809266 & 2.809266 \\
\hline \multicolumn{10}{|c|}{ 4-component } \\
\hline DHF & -100.16257387 & & & & & & & & 2.809754 \\
\hline
\end{tabular}

pointed out by Malkin et al. ${ }^{20}$ a first-order property transformation, i.e., the free-particle Foldy-Wouthuysen transformation, contributes to a large extent to the correction for the picture-change effect. Higher-order terms decline rapidly with increasing order. The standard $\operatorname{DKH}(2,2)$ approach can be regarded as a suitable choice for lighter elements including iodine, where the deviation from a $\operatorname{DKH}(7,7) \mathrm{EFG}$ data adds up to 0.07 a.u., which is smaller than the computational error introduced by the finite basis set. For At already $\operatorname{DKH}(4,4)$ offers a fast and accurate alternative.

Spin-orbit effects are small for the closed-shell species under study, excepting HAt where spin-orbit coupling tends to diminish the EFG by some 0.5 a.u. An explanation for that was given by Pyykkö and Seth by addressing the so-called spin-orbit tilting effect. ${ }^{34}$ For the lighter homologs, however, the $\operatorname{DKH}(7,7)$ results do not deviate much from the DHF ones.

\section{B. Effects of a finite-nucleus model on the EFG}

Tables III-VII also provide the results for the EFG of the set of HX molecules within different $\operatorname{DKH}(n, m)$ models, as obtained with a finite nuclear charge distribution. The Gaussian charge distribution model of Visscher and Dyall was chosen to represent the nucleus in our study. ${ }^{35}$ The very same finite-nucleus model exponents were also applied in all fourcomponent calculations. Switching from a point charge nucleus to a finite-nucleus model does not show a significant

TABLE IV. Electronic energy and principal component of the diagonalized electric field gradient tensor $\left(q_{z z}\right)$ in $\mathrm{HCl}$ (in a.u.) calculated at the $\mathrm{Cl}$ nucleus.

\begin{tabular}{|c|c|c|c|c|c|c|c|c|c|}
\hline $\mathrm{DKH} n$ & Energy & $q_{z z}(n, 0)$ & $q_{z z}(n, 1)$ & $q_{z z}(n, 2)$ & $q_{z z}(n, 3)$ & $q_{z z}(n, 4)$ & $q_{z z}(n, 5)$ & $q_{z z}(n, 6)$ & $q_{z z}(n, 7)$ \\
\hline \multicolumn{10}{|c|}{ Point charge nucleus/Optimum parametrization } \\
\hline Nonrel & -460.11266042 & 3.541586 & & & & & & & \\
\hline DKH2 & -461.52294727 & 3.619627 & 3.590193 & 3.590205 & & & & & \\
\hline DKH3 & -461.52629083 & 3.619629 & 3.590195 & 3.590207 & 3.590207 & & & & \\
\hline DKH4 & -461.52618704 & 3.619629 & 3.590195 & 3.590207 & 3.590207 & 3.590207 & & & \\
\hline DKH5 & -461.52619558 & 3.619629 & 3.590195 & 3.590207 & 3.590207 & 3.590207 & 3.590207 & & \\
\hline DKH6 & -461.52619483 & 3.619629 & 3.590195 & 3.590207 & 3.590207 & 3.590207 & 3.590207 & 3.590207 & \\
\hline DKH7 & -461.52619489 & 3.619629 & 3.590195 & 3.590207 & 3.590207 & 3.590207 & 3.590207 & 3.590207 & 3.590207 \\
\hline \multicolumn{10}{|c|}{ Finite nucleus/Optimum parametrization } \\
\hline Nonrel & -460.11221460 & 3.541586 & & & & & & & \\
\hline DKH2 & -461.52246883 & 3.619626 & 3.590191 & 3.590204 & & & & & \\
\hline DKH3 & -461.52579534 & 3.619628 & 3.590193 & 3.590205 & 3.590205 & & & & \\
\hline DKH4 & -461.52569287 & 3.619628 & 3.590193 & 3.590205 & 3.590205 & 3.590205 & & & \\
\hline DKH5 & -461.52570122 & 3.619628 & 3.590193 & 3.590205 & 3.590205 & 3.590205 & 3.590205 & & \\
\hline DKH6 & -461.52570050 & 3.619628 & 3.590193 & 3.590205 & 3.590205 & 3.590205 & 3.590205 & 3.590205 & \\
\hline DKH7 & -461.52570055 & 3.619628 & 3.590193 & 3.590205 & 3.590205 & 3.590205 & 3.590205 & 3.590205 & 3.590205 \\
\hline \multicolumn{10}{|c|}{ 4-component } \\
\hline DHF & -461.56845082 & & & & & & & & 3.591314 \\
\hline
\end{tabular}


TABLE V. Electronic energy and principal component of the diagonalized electric field gradient tensor $\left(q_{z z}\right)$ in $\mathrm{HBr}$ (in a.u.) calculated at the Br nucleus.

\begin{tabular}{|c|c|c|c|c|c|c|c|c|c|}
\hline $\mathrm{DKH} n$ & Energy & $q_{z z}(n, 0)$ & $q_{z z}(n, 1)$ & $q_{z z}(n, 2)$ & $q_{z z}(n, 3)$ & $q_{z z}(n, 4)$ & $q_{z z}(n, 5)$ & $q_{z z}(n, 6)$ & $q_{z z}(n, 7)$ \\
\hline \multicolumn{10}{|c|}{ Point charge nucleus/Optimum parametrization } \\
\hline Nonrel & -2573.05225597 & 7.009477 & & & & & & & \\
\hline DKH2 & -2604.91195760 & 7.840219 & 7.537429 & 7.536201 & & & & & \\
\hline DKH3 & -2605.12205555 & 7.840417 & 7.537597 & 7.536368 & 7.536551 & & & & \\
\hline DKH4 & -2605.10839261 & 7.840403 & 7.537585 & 7.536356 & 7.536539 & 7.536520 & & & \\
\hline DKH5 & -2605.11085654 & 7.840405 & 7.537587 & 7.536358 & 7.536541 & 7.536522 & 7.536524 & & \\
\hline DKH6 & -2605.11039436 & 7.840404 & 7.537586 & 7.536358 & 7.536540 & 7.536521 & 7.536523 & 7.536523 & \\
\hline DKH7 & -2605.11046752 & 7.840404 & 7.537586 & 7.536358 & 7.536540 & 7.536521 & 7.536523 & 7.536523 & 7.536523 \\
\hline \multicolumn{10}{|c|}{ Finite nucleus/Optimum parametrization } \\
\hline Nonrel & -2573.03894388 & 7.009468 & & & & & & & \\
\hline DKH2 & -2604.89427463 & 7.840026 & 7.537244 & 7.536047 & & & & & \\
\hline DKH3 & -2605.10163419 & 7.840222 & 7.537410 & 7.536213 & 7.536390 & & & & \\
\hline DKH4 & -2605.08839257 & 7.840208 & 7.537398 & 7.536201 & 7.536378 & 7.536360 & & & \\
\hline DKH5 & -2605.09071133 & 7.840210 & 7.537400 & 7.536203 & 7.536380 & 7.536362 & 7.536364 & & \\
\hline DKH6 & -2605.09029540 & 7.840209 & 7.537399 & 7.536203 & 7.536380 & 7.536361 & 7.536363 & 7.536363 & \\
\hline DKH7 & -2605.09035788 & 7.840209 & 7.537399 & 7.536203 & 7.536380 & 7.536361 & 7.536363 & 7.536363 & 7.536363 \\
\hline \multicolumn{10}{|c|}{ 4-component } \\
\hline DHF & -2605.63312416 & & & & & & & & 7.540289 \\
\hline
\end{tabular}

effect in case of all molecules with the exception of HAt, where a finite nucleus accounts for a decrease of the EFG by 0.18 a.u. EFG calculations on heavy elements are thus sensitive to the type of nucleus chosen, i.e., whether it features the singular attraction potential of a point charge (with its well-known effect on the short-range expansion of the spherically averaged spinor) or the finite potential of an extended charge distribution. ${ }^{36}$ For heavier atoms than At, the effect of a finite nuclear charge distribution can be expected to be even larger. Therefore, a careful choice of the nucleus model chosen might be a crucial issue in calculations on heavy and superheavy atoms. It is likely that different finitenucleus models such as a homogeneously charged sphere or a Fermi nucleus will not change the qualitative picture obtained from the Gaussian model (compare to the results of Ref. 35).

\section{EFG calculated with standard basis sets}

Since basis sets of sizes as used in the present study are unfeasible for standard applications, we repeated the $\operatorname{DKH}(2,2)$ and $\operatorname{DKH}(4,3)$ calculations on the whole hydrogen halide series with ANO-RCC (Ref. 37) basis sets, as implemented in MOLCAS. These generally contracted basis sets were especially derived for scalar-relativistic one-component calculations by using a second-order DKH Hamiltonian.

TABLE VI. Electronic energy and principal component of the diagonalized electric field gradient tensor $\left(q_{z z}\right)$ in HI (in a.u.) calculated at the I nucleus.

\begin{tabular}{|c|c|c|c|c|c|c|c|c|c|}
\hline $\mathrm{DKH} n$ & Energy & $q_{z z}(n, 0)$ & $q_{z z}(n, 1)$ & $q_{z z}(n, 2)$ & $q_{z z}(n, 3)$ & $q_{z z}(n, 4)$ & $q_{z z}(n, 5)$ & $q_{z z}(n, 6)$ & $q_{z z}(n, 7)$ \\
\hline \multicolumn{10}{|c|}{ Point charge nucleus/Optimum parametrization } \\
\hline Nonrel & -6918.57486707 & 9.670500 & & & & & & & \\
\hline DKH2 & -7111.45929389 & 12.873105 & 11.655929 & 11.618554 & & & & & \\
\hline DKH3 & -7113.62730438 & 12.874893 & 11.657317 & 11.619930 & 11.628742 & & & & \\
\hline DKH4 & -7113.41267221 & 12.874679 & 11.657134 & 11.619748 & 11.628560 & 11.626853 & & & \\
\hline DKH5 & -7113.47581188 & 12.874721 & 11.657173 & 11.619787 & 11.628600 & 11.626892 & 11.627224 & & \\
\hline DKH6 & -7113.45720105 & 12.874707 & 11.657162 & 11.619776 & 11.628589 & 11.626881 & 11.627213 & 11.627152 & \\
\hline DKH7 & -7113.46160943 & 12.874710 & 11.657165 & 11.619778 & 11.628591 & 11.626884 & 11.627216 & 11.627155 & 11.627166 \\
\hline \multicolumn{10}{|c|}{ Finite nucleus/Optimum parametrization } \\
\hline Nonrel & -6918.48082583 & 9.670452 & & & & & & & \\
\hline DKH2 & -7111.27574193 & 12.857503 & 11.643874 & 11.614194 & & & & & \\
\hline DKH3 & -7113.38809120 & 12.859200 & 11.645247 & 11.615560 & 11.622568 & & & & \\
\hline DKH4 & -7113.18513862 & 12.858992 & 11.645080 & 11.615393 & 11.622401 & 11.621046 & & & \\
\hline DKH5 & -7113.24155767 & 12.859028 & 11.645115 & 11.615428 & 11.622437 & 11.621081 & 11.621348 & & \\
\hline DKH6 & -7113.22611820 & 12.859016 & 11.645106 & 11.615419 & 11.622427 & 11.621072 & 11.621338 & 11.621288 & \\
\hline DKH7 & -7113.22947346 & 12.859019 & 11.645108 & 11.615421 & 11.622429 & 11.621074 & 11.621340 & 11.621290 & 11.621299 \\
\hline \multicolumn{10}{|c|}{ 4-component } \\
\hline DHF & -7116.38613382 & & & & & & & & 11.623332 \\
\hline
\end{tabular}


TABLE VII. Electronic energy and principal component of the diagonalized electric field gradient tensor $\left(q_{z z}\right)$ in HAt (in a.u.) calculated at the At nucleus.

\begin{tabular}{|c|c|c|c|c|c|c|c|c|c|}
\hline $\mathrm{DKH} n$ & Energy & $q_{z z}(n, 0)$ & $q_{z z}(n, 1)$ & $q_{z z}(n, 2)$ & $q_{z z}(n, 3)$ & $q_{z z}(n, 4)$ & $q_{z z}(n, 5)$ & $q_{z z}(n, 6)$ & $q_{z z}(n, 7)$ \\
\hline \multicolumn{10}{|c|}{ Point charge nucleus/Optimum parametrization } \\
\hline Nonrel & -21267.46707151 & 15.358363 & & & & & & & \\
\hline DKH2 & -22846.39360406 & 37.171665 & 27.425119 & 26.409692 & & & & & \\
\hline DKH3 & -22877.97182332 & 37.200149 & 27.446665 & 26.430743 & 26.782486 & & & & \\
\hline DKH4 & -22873.13085658 & 37.194075 & 27.441024 & 26.425127 & 26.776879 & 26.659425 & & & \\
\hline DKH5 & -22875.91807419 & 37.197022 & 27.442715 & 26.426715 & 26.778503 & 26.661037 & 26.699892 & & \\
\hline DKH6 & -22874.50288735 & 37.195213 & 27.441822 & 26.425900 & 26.777661 & 26.660204 & 26.699056 & 26.686713 & \\
\hline DKH7 & -22875.00564174 & 37.195680 & 27.442169 & 26.426249 & 26.778012 & 26.660555 & 26.699407 & 26.687064 & 26.690805 \\
\hline \multicolumn{10}{|c|}{ Finite nucleus/Optimum parametrization } \\
\hline Nonrel & -21266.61858632 & 15.358042 & & & & & & & \\
\hline DKH2 & -22840.66459810 & 36.391780 & 26.978222 & 26.335521 & & & & & \\
\hline DKH3 & -22869.97635286 & 36.416796 & 26.997685 & 26.354736 & 26.572096 & & & & \\
\hline DKH4 & -22865.65850892 & 36.410276 & 26.993094 & 26.350251 & 26.567579 & 26.497028 & & & \\
\hline DKH5 & -22867.80961248 & 36.412034 & 26.994367 & 26.351501 & 26.568836 & 26.498282 & 26.521140 & & \\
\hline DKH6 & -22866.84160469 & 36.411008 & 26.993782 & 26.350940 & 26.568267 & 26.497716 & 26.520573 & 26.513359 & \\
\hline DKH7 & -22867.14164046 & 36.411262 & 26.993964 & 26.351129 & 26.568457 & 26.497906 & 26.520763 & 26.513548 & 26.515799 \\
\hline \multicolumn{10}{|c|}{ 4-component } \\
\hline DHF & -22910.39450051 & & & & & & & & 25.979844 \\
\hline
\end{tabular}

They might, however, not offer an optimal description of core orbitals in heavy elements, when higher-order DKH transformations are involved. We chose the $\operatorname{DKH}(2,2)$ and $\operatorname{DKH}(4,3)$ approaches for this comparison because both do not depend on the parametrization of the unitary matrices. While $\operatorname{DKH}(2,2)$ calculations represent the "standard" DKH model, $\operatorname{DKH}(4,3)$ provides the highest order possible if a dependence on the parametrization of the unitary matrices shall be avoided. As can be seen from Table IX, reliable values for the EFG are obtained even with basis sets of modest size. For the two heaviest elements the error due to the incompleteness of the basis set is $1 \%-2 \%$. Thus for $\mathrm{Br}$ and its heavier homologs, the error introduced by the picturechange effect is by far larger than the inaccuracies owing to the limited basis set size and has to be considered explicitly.

\section{Dependence of the EFG on the parametrization of the unitary transformation}

Different parametrization schemes characterized by different sets of expansion coefficients $a_{m, j}$ of Eq. (7) do not have significant impact on the calculation of the EFG, as can be demonstrated on the basis of Table X. We chose two different models $\operatorname{DKH}(5,4)$ and $\operatorname{DKH}(6,6)$ in order to demonstrate the magnitude of this effect as depending on the DKH order.

TABLE VIII. Percental scalar-relativistic effect on $q_{z z}$ and relative picturechange error as defined by Eq. (14).

\begin{tabular}{ccc}
\hline \hline Molecule & Scalar-relativistic effect on $q_{z z}(\%)$ & PCE $_{\text {rel }}(\%)$ \\
\hline $\mathrm{HF}$ & 0.3 & 0.2 \\
$\mathrm{HCl}$ & 0.4 & 0.8 \\
$\mathrm{HBr}$ & 7 & 4 \\
$\mathrm{HI}$ & 17 & 11 \\
$\mathrm{HAt}$ & 41 & 37 \\
\hline \hline
\end{tabular}

The five different parametrization schemes compared for $\operatorname{DKH}(6,6)$ taking the heavy hydrogen halides HI and HAt as examples reveal an absolute dependence of the EFG on the parametrization of the order of magnitude less than $10^{-4}$ a.u., which can be below the accuracy of the electronic structure method. The same holds for the $\operatorname{DKH}(5,4)$ calculations where the largest parameter dependence is expected. Thus the choice of parametrization affects the electronic energy to a greater extent than the molecular property in this example.

\section{E. Comparison to data from the literature}

A sensible comparison of our results to data from the literature on the EFG in the hydrogen halide series is only appropriate for the data given by Malkin et al..$^{20}$ and Neese et al. ${ }^{21}$ because they both provide results obtained with an analytical treatment of the DKH property transformation. Since only the electronic contribution to the EFG was published by Malkin et al., we have evaluated the missing nuclear contribution and complemented the original data set. In addition, we compare to the finite-field results of Visscher et al. ${ }^{30}$ which depend on this numerical model but incorporate electron correlation effects. The corresponding data can be found in Table XI.

First of all we should like to draw attention to the deviation of results obtained with the forward and back transformation schemes in the cases of HI and HAt in Ref. 21. For these two heaviest elements, our results do not confirm the back transformation results of Ref. 21, but show that our back transformation approach agrees well with the DHF reference as it should be [compare Eq. (5)]. The agreement is even better in the case of HAt if we contrast the $\operatorname{DKH}(2,2)$ EFG of 26.409692 a.u. with the spin-free DHF EFG of 26.588178 a.u. (see Ref. 38 for this spin-free variant of DHF). On the contrary, it is surprising to note that the electric-field-dependent forward transformation agrees well with our results and DHF. In most cases our results are 
TABLE IX. Electronic energy and principal component of the diagonalized electric field gradient tensor $\left(q_{z z}\right)$ in the halogen halide series (in a.u.) calculated with a $\operatorname{DKH}(2,2)$ and $\operatorname{DKH}(4,3)$ formalism at the halogen nucleus with standard ANO-RCC basis sets (Ref. 37). Relative differences (in \%) to the $\operatorname{DKH}(2,2)$ and $\operatorname{DKH}(4,3)$ values obtained with large decontracted basis sets are given for comparison.

\begin{tabular}{|c|c|c|c|c|c|}
\hline Molecule & Energy & $\operatorname{DKH}(n, m)$ & $q_{z z}(n, m)$ & $\Delta q_{z z}(n, m)(\%)$ & Basis set (heavy element) ${ }^{\mathrm{a}}$ \\
\hline \multirow[t]{2}{*}{$\mathrm{HF}$} & -100.15713804 & $(2,2)$ & 2.867641 & 2.1 & \multirow[t]{2}{*}{$14 s 9 p 4 d 3 f 2 g / 8 s 7 p 4 d 3 f 2 g$} \\
\hline & -100.15719910 & $(4,3)$ & 2.867645 & 2.1 & \\
\hline \multirow[t]{2}{*}{$\mathrm{HCl}$} & -461.52114815 & $(2,2)$ & 3.631357 & 1.1 & \multirow[t]{2}{*}{$17 s 12 p 5 d 4 f 2 g / 8 s 7 p 5 d 4 f 2 g$} \\
\hline & -461.52410133 & $(4,3)$ & 3.631356 & 1.1 & \\
\hline \multirow[t]{2}{*}{$\mathrm{HBr}$} & -2604.90959142 & $(2,2)$ & 7.503138 & 0.4 & \multirow[t]{2}{*}{$20 s 17 p 11 d 4 f 2 g / 9 s 8 p 6 d 4 f 2 \varepsilon$} \\
\hline & -2605.10499775 & $(4,3)$ & 7.503860 & 0.4 & \\
\hline \multirow[t]{2}{*}{$\mathrm{HI}$} & -7111.44901584 & $(2,2)$ & 11.486758 & 1.1 & \multirow[t]{2}{*}{$22 s 19 p 13 d 5 f 3 g / 10 s 9 p 8 d 5 f 3 g$} \\
\hline & -7113.39201977 & $(4,3)$ & 11.493198 & 1.2 & \\
\hline \multirow[t]{2}{*}{ HAt } & -22846.01272439 & $(2,2)$ & 26.137657 & 1.0 & \multirow[t]{2}{*}{$25 s 22 p 16 d 12 f 4 g / 11 s 10 p 9 d 6 f 4 g$} \\
\hline & -22872.40512985 & $(4,3)$ & 26.383652 & 1.5 & \\
\hline
\end{tabular}

${ }^{\mathrm{a}}$ Reference 37.
${ }^{\mathrm{b}}$ Reference 39.

smaller than those published by Neese et al., ${ }^{21}$ Malkin et al. ${ }^{20}$ and Visscher et al. ${ }^{30}$ This is likely to be due to the additional tight polarization functions in our work. Steep functions of higher angular momentum hardly contribute to the electronic energy of the molecules under investigation, but they tend to diminish the expectation value of the EFG. This effect was also observed by Visscher et al..$^{30}$ The basis sets used in Ref. 20 are, in general, considerably smaller than those employed in the other studies under consideration. This

TABLE X. Electronic energy and principal component of the diagonalized electric field gradient tensor $\left(q_{z z}\right)$ in HI and HAt (in a.u.) calculated with the $\operatorname{DKH}(6,6)$ and $\operatorname{DKH}(5,4)$ protocols at the I and At nucleus, using different parametrization schemes. These schemes provide different sets of expansion coefficients $\left\{a_{m, j}\right\}$ given in Ref. 5. Note that the energy is classified by $n$ and hence either of sixth or fifth order. The fifth order is below the sixth order following the oscillatory convergence in Ref. 10.

\begin{tabular}{rccc}
\hline \hline Parametrization & DKH $(n, m)$ & Energy & $q_{z z}(n, m)$ \\
\hline & & HI & \\
Cayley & $(6,6)$ & -7113.45717625 & 11.627143 \\
& $(5,4)$ & -7113.47604696 & 11.626883 \\
Exponential & $(6,6)$ & -7113.45719621 & 11.627150 \\
& $(5,4)$ & -7113.47585778 & 11.626891 \\
McWeeny & $(6,6)$ & -7113.45711638 & 11.627120 \\
& $(5,4)$ & -7113.47661451 & 11.626860 \\
Optimum & $(6,6)$ & -7113.45720105 & 11.627152 \\
& $(5,4)$ & -7113.47581188 & 11.626892 \\
Square root & $(6,6)$ & -7113.45723614 & 11.627166 \\
& $(5,4)$ & -7113.47547944 & 11.626906 \\
& & & \\
Cayley & $(6,6)$ & -22874.49955311 & 26.686711 \\
& $(5,4)$ & -22875.94206758 & 26.661039 \\
Exponential & $(6,6)$ & -22874.50223624 & 26.686713 \\
& $(5,4)$ & -22875.92275882 & 26.661029 \\
McWeeny & $(6,6)$ & -22874.49150627 & 26.686705 \\
& $(5,4)$ & -22876.00001244 & 26.661067 \\
Optimum & $(6,6)$ & -22874.50288735 & 26.686713 \\
& $(5,4)$ & -22875.91807419 & 26.661037 \\
Square root & $(6,6)$ & -22874.50760381 & 26.686715 \\
& $(5,4)$ & -22875.88415057 & 26.661010 \\
\hline \hline
\end{tabular}

gives rise to somewhat larger deviations when comparing our results to the data provided by Ref. 20, especially in case of the EFG at the fluorine nucleus in $\mathrm{HF}$ and the chlorine nucleus in $\mathrm{HCl}$. Note that if electron correlation is taken into account, a change of about $7 \%$ for the EFG in $\mathrm{HI}$ was estimated by Visscher et al. ${ }^{30}$

\section{CONCLUSION}

Quantum chemical calculations of energies and molecular properties on molecules containing heavy elements require a consistent treatment of relativistic effects. For the closed-shell molecules under investigation spin-orbit effects can be shown to be rather small, excluding HAt, where they diminish the expectation value of the EFG by more than 0.5 a.u. A scalar-relativistic treatment is thus sufficient for all studied molecules with the exception of HAt. A DKH $(2,2)$ calculation, which may be considered the standard approach for the analytical determination of the electric field gradient on molecules up to a nuclear charge of $Z \approx 50$, provides reliable results within the accuracy of the computational method chosen. A complete neglect of the property transformation is in no case acceptable because the error introduced by the picture-change effect leads to an overestimation of the $\mathrm{EFG}$, which lies in the order of magnitude of the relativistic corrections. For heavier elements a $\operatorname{DKH}(3,3)$ or even higher-order schemes can be regarded as an appropriate choice for performing scalar-relativistic calculations. Moreover, the choice of the nuclear charge distribution model affects the calculation of the electric field gradient with increased nuclear charge $Z$ and should be considered, especially for heavy atoms. However, the errors introduced due to the incompleteness of standard basis set can be far larger than the corrections to the EFG provided by higherorder property transformations. Hence time and memoryconsuming high-order corrections in case of a conventional DKH implementation are out of all proportion to the gain in 
TABLE XI. Principal component of the diagonalized electric field gradient tensor $\left(q_{z z}\right)$ in the hydrogen halides series HX (in a.u.) at the halogen nucleus compared to EFG values calculated by Neese et al. (Ref. 21), Malkin et al. (Ref. 20), and Visscher et al. (Ref. 30). Bond distances and basis sets are given for comparison. (FT)/(BT) refer to "forward transformation" and "back transformation," respectively. The "basis" is the uncontracted basis set at the halogen atom and the bond distance (in bohrs) is denoted as $d$. All four-component DHF and CCSD(T) [denoted as 4-c-CCSD $(\mathrm{T})$ ] calculations were performed with a Gaussian nuclear charge distribution, while the $\operatorname{DKH}(2,2)$ results were obtained with a point-charge nucleus model.

\begin{tabular}{|c|c|c|c|c|}
\hline & Neese et al. & Malkin et al. & Visscher et al. & This work \\
\hline \multicolumn{5}{|c|}{$\mathrm{HF}$} \\
\hline$d$ & 1.732549 & 1.733 & 1.7328 & 1.732549 \\
\hline Basis & $23 s / 16 p / 3 d / 1 f$ & $9 s / 5 p / 1 d$ & aug-cc-pVTZ & $23 s / 17 p / 6 d / 4 f$ \\
\hline 4-c-CCSD(T) & $\ldots$ & $\ldots$ & 2.696 & $\ldots$ \\
\hline DHF & 2.881 & $\cdots$ & 2.870 & 2.809754 \\
\hline HF DKH $(2,2)$ & $\begin{array}{l}2.8799(\mathrm{BT}) \\
2.8801(\mathrm{FT})\end{array}$ & 2.963 & $\cdots$ & $2.809265(\mathrm{BT})$ \\
\hline \multicolumn{5}{|c|}{$\mathrm{HCl}$} \\
\hline$d$ & 2.408502 & 2.409 & 2.409 & 2.408502 \\
\hline Basis & $26 s / 19 p / 3 d / 1 f$ & $11 s / 7 p / 2 d$ & aug-cc-pVTZ & $24 s / 19 p / 7 d / 5 f$ \\
\hline 4-c-CCSD(T) & $\ldots$ & $\cdots$ & 3.377 & $\cdots$ \\
\hline DHF & 3.660 & $\cdots$ & 3.638 & 3.591314 \\
\hline $\mathrm{HF}[\mathrm{DKH}(2,2)]$ & $\begin{array}{l}3.6561(\mathrm{BT}) \\
3.6587(\mathrm{FT})\end{array}$ & 3.803 & $\cdots$ & 3.590205 (BT) \\
\hline \multicolumn{5}{|c|}{$\mathrm{HBr}$} \\
\hline$d$ & 2.672946 & 2.673 & 2.673 & 2.672946 \\
\hline Basis & $29 s / 23 p / 16 d / 3 f$ & $15 s / 12 p / 7 d / 2 f$ & $23 s / 16 p / 12 d / 3 f$ & $30 s / 22 p / 14 d / 8 f / 3 g$ \\
\hline 4-c-CCSD(T) & $\cdots$ & $\cdots$ & 7.035 & $\cdots$ \\
\hline DHF & 7.602 & $\ldots$ & 7.603 & 7.540289 \\
\hline $\operatorname{HF} \operatorname{DKH}(2,2)$ & $\begin{array}{l}7.5365(\mathrm{BT}) \\
7.5998(\mathrm{FT})\end{array}$ & 7.560 & $\cdots$ & 7.536201 (BT) \\
\hline \multicolumn{5}{|c|}{ HI } \\
\hline$d$ & 3.040798 & 3.040 & 3.040 & 3.040798 \\
\hline Basis & $31 s / 26 p / 19 d / 3 f$ & $17 s / 13 p / 9 d / 2 f$ & $27 s / 21 p / 15 d / 4 f$ & $35 s / 28 p / 19 d / 8 f / 4 g$ \\
\hline 4-c-CCSD(T) & $\ldots$ & $\ldots$ & 10.751 & $\ldots$ \\
\hline DHF & 11.679 & $\ldots$ & 11.658 & 11.623332 \\
\hline $\operatorname{HF} \mathrm{DKH}(2,2)$ & $\begin{array}{l}11.2901(\mathrm{BT}) \\
11.7004(\mathrm{FT})\end{array}$ & 11.560 & $\cdots$ & 11.618554 (BT) \\
\hline \multicolumn{5}{|c|}{ HAt } \\
\hline$d$ & 3.250392 & $\cdots$ & $\cdots$ & 3.250392 \\
\hline Basis & $31 s / 27 p / 21 d / 15 f$ & $\cdots$ & $\cdots$ & $37 s / 32 p / 22 d / 15 f / 4 g$ \\
\hline 4-c-CCSD(T) & $\ldots$ & $\cdots$ & $\cdots$ & $\ldots$ \\
\hline DHF & 25.976 & $\cdots$ & $\cdots$ & 25.979844 \\
\hline $\operatorname{HF} \operatorname{DKH}(2,2)$ & $\begin{array}{l}21.4231(\mathrm{BT}) \\
26.9665(\mathrm{FT})\end{array}$ & $\cdots$ & $\cdots$ & 26.409692 (BT) \\
\hline
\end{tabular}

accuracy. The choice of the parametrization scheme for the unitary matrices in the DKH approach does hardly affect the expectation value of the EFG, and has thus not to be considered when setting up a calculation. While the standard $\operatorname{DKH}(2,2)$ model is likely to perform well for most cases, we may recommend the $\operatorname{DKH}(4,3)$ model, which is still independent of the chosen parametrization, as valid for the whole of the Periodic Table. In conclusion, we should emphasize that the field-independent unitary transformation is the appropriate approach to match results from four-component first-order perturbation theory. In standard DKH calculations, however, one neglects all spin-dependent terms which then, of course, introduces additional sources of error.

\section{ACKNOWLEDGMENTS}

The authors thank the Schweizer National Fonds (Project no. 200021-113479/1) and the Swedish Research Council (VR) for financial support.

${ }^{1}$ P. Schwerdtfeger, Relativistic Quantum Chemistry-Part I. Fundamentals, Theoretical and Computational Chemistry (Elsevier, Amsterdam, 2002).

${ }^{2}$ P. Schwerdtfeger, Relativistic Quantum Chemistry-Part II. Applications, Theoretical and Computational Chemistry (Elsevier, Amsterdam, 2004).

${ }^{3}$ M. Douglas and N. M. Kroll, Ann. Phys. 82, 89 (1974).

${ }^{4}$ B. A. Hess, Phys. Rev. A 33, 3742 (1986).

${ }^{5}$ A. Wolf, M. Reiher, and B. A. Hess, J. Chem. Phys. 117, 9215 (2002).

${ }^{6}$ M. Reiher, Theor. Chem. Acc. 116, 241 (2006).

${ }^{7}$ T. Nakajima and K. Hirao, J. Chem. Phys. 113, 7786 (2000).

${ }^{8}$ C. van Wüllen, J. Chem. Phys. 120, 7307 (2004). 
${ }^{9}$ M. Reiher and A. Wolf, J. Chem. Phys. 121, 2037 (2004).

${ }^{10}$ M. Reiher and A. Wolf, J. Chem. Phys. 121, 10945 (2004).

${ }^{11}$ E. J. Baerends, W. H. E. Schwarz, P. Schwerdtfeger, and J. G. Snijders, J. Phys. B 23, 3225 (1990).

${ }^{12}$ V. Kellö and A. J. Sadlej, Int. J. Quantum Chem. 68, 159 (1998).

${ }^{13}$ M. Pernpointer, P. Schwerdtfeger, and B. A. Hess, Int. J. Quantum Chem. 76, 371 (2000).

${ }^{14}$ V. Kellö, Mol. Phys. 89, 127 (1996).

${ }^{15}$ O. Visser, L. Visscher, P. J. C. Aerts, and W. C. Nieuwpoort, Theor. Chim. Acta 81, 405 (1992).

${ }^{16}$ P. Schwerdtfeger, M. Pernpointer, and W. Nazarewicz, in Calculation of NMR and EPR Parameters: Theory and Applications, edited by M. Kaupp, M. Bühl, and V. G. Malkin (Wiley-VCH, Weinheim, 2004), pp. 279-291.

${ }^{17}$ J. W. Krogh, G. Barone, and R. Lindh, Chem.-Eur. J. 12, 5116 (2006).

${ }^{18}$ M. Pernpointer, M. Seth, and P. Schwerdtfeger, J. Chem. Phys. 108, $6722(1998)$

${ }^{19}$ V. Kellö and A. J. Sadlej, J. Chem. Phys. 120, 9424 (2004).

${ }^{20}$ I. Malkin, O. L. Malkina, and V. G. Malkin, Chem. Phys. Lett. 361, 231 (2002).

${ }^{21}$ F. Neese, A. Wolf, T. Fleig, M. Reiher, and B. A. Hess, J. Chem. Phys. 122, 204107 (2005).

${ }^{22}$ F. Schwabl, Advanced Quantum Mechanics, 3rd ed. (Springer-Verlag, Berlin, 2005).
${ }^{23}$ A. Wolf and M. Reiher, J. Chem. Phys. 124, 064102 (2006).

${ }^{24}$ A. Wolf and M. Reiher, J. Chem. Phys. 124, 064103 (2006).

${ }^{25}$ M. Reiher and A. Wolf, Phys. Lett. A 360, 603 (2007).

${ }^{26}$ I. Malkin, O. L. Malkina, V. G. Malkin, and M. Kaupp, Chem. Phys. Lett. 396, 268 (2004).

${ }^{27}$ I. Malkin, Ph.D. thesis, University of Würzburg, 2006

${ }^{28}$ G. Karlström, R. Lindh, P.-Å. Malmqvist et al., Comput. Mater. Sci. 28, 222 (2003).

${ }^{29}$ H. J. Jensen, T. Saue, L. Visscher et al., DIRAC 04, a relativistic ab initio electronic structure program, Release DIRAC04.0, http://dirac.chem.sdu.dk

${ }^{30}$ L. Visscher, T. Enevoldsen, T. Saue, and J. Oddershede, J. Chem. Phys. 109, 9677 (1998).

${ }^{31}$ K. G. Dyall, Theor. Chem. Acc. 115, 441 (2006).

${ }^{32}$ D. Woon and T. H. Dunning, J. Chem. Phys. 100, 2975 (1994).

${ }^{33}$ A. Wilson, T. van Mourik, and T. H. Dunning, J. Mol. Struct.: THEOCHEM 388, 339 (1997).

${ }^{34}$ P. Pyykkö and M. Seth, Theor. Chem. Acc. 96, 92 (1997).

${ }^{35}$ L. Visscher and K. G. Dyall, At. Data Nucl. Data Tables 67, 207 (1997).

${ }^{36}$ D. Andrae, Phys. Rep. 336, 413 (2000).

${ }^{37}$ B. O. Roos, R. Lindh, P.-Å. Malmqvist, V. Veryazov, and P.-O. Widmark, J. Phys. Chem. A 108, 2851 (2005).

${ }^{38}$ K. G. Dyall, J. Chem. Phys. 100, 2118 (1994).

${ }^{39}$ P.-O. Widmark, P.-Å. Malmqvist, and B. O. Roos, Theor. Chim. Acta 77, 291 (1990). 